SNUST-000901

hep-th/0012165

\title{
Global Black p-Brane World: A New Approach to Stable Mass Hierarchy t
}

\author{
Sei-Hoon Moon ${ }^{a}$, Soo-Jong Rey ${ }^{a}$ and Yoonbai Kim ${ }^{b}$ \\ School of Physics \&3 Center for Theoretical Physics \\ Seoul National University, Seoul 151-742 Korea ${ }^{a}$ \\ Department of Physics 83 Institute of Basic Science \\ Sungkyunkwan University, Suwon 440-746 Korea ${ }^{b}$ \\ jeollo@phya.snu.ac.kr, sjrey@gravity.snu.ac.kr, yoonbai@cosmos.skku.ac.kr
}

\begin{abstract}
We find a class of extremal black hole-like global $p$-brane in higher-dimensional gravity with a negative cosmological constant. The region inside the $p$-brane horizon possesses all essential features required for the Randall-Sundrum-type brane world scenario. The set-up allows to interpret the horizon size as the compactification size in that the Planck scale $M_{\mathrm{P} 1}$ is determined by the fundamental scale $M_{*}$ and the horizon size $r_{\mathrm{H}}$ via the familiar relation $M_{\mathrm{Pl}}^{2} \sim M_{*}^{2+n} r_{\mathrm{H}}^{n}$, and the gravity behaves as expected in a world with $n$-extra dimensions compactified with size $r_{\mathrm{H}}$. Most importantly, a stable mass hierarchy between $M_{\mathrm{Pl}}$ and $M_{*}$ can be generated from topological charge of the $p$-brane and the horizon size $r_{\mathrm{H}}$ therein. We also offer a new perspective on various issues associated to the brane world scenarios including the cosmological constant problem.
\end{abstract}

\footnotetext{
${ }^{1}$ Work supported in part by BK-21 Initiative in Physics (SNU - Project 2), KRF International Collaboration Grant 1998-010-192, KOSEF Interdisciplinary Research Grant 98-07-02-07-01-5, and KOSEF Leading Scientist Program 2000-1-11200-001-1 (S.-J. R.) and KOSEF Basic Research Program No. 2000-1-11200-001-3 (Y. K.).
} 


\section{Introduction}

In exploring brane world scenarios in recent years, seemingly two alternative directions have been pursued.

First direction, as pioneered by Arkani-Hamed, Dimopoulos and Dvali (ADD), involves reducing the fundamental mass scale from the four-dimensional Planck scale, $M_{\mathrm{Pl}} \sim 10^{19} \mathrm{GeV}$, to the electroweak scale, $m_{\mathrm{EW}} \sim 10^{3} \mathrm{GeV}$, by introducing large but compact extra dimensions. Current experimental bound suggests that the extra dimensions could be as big as sub-millimeter scale when the extra dimensions are two [1]. In the simple examples of [1], 2, [3] proposal, the spacetime geometry is a direct product of four-dimensional Minkowski spacetime and a spatial $n$-torus of volume $\mathrm{Vol}_{n} \sim R^{n}$. Then, at low-energy, effective four-dimensional Planck scale $M_{\mathrm{Pl}}$ is set by the Gauss' relation, $M_{\mathrm{Pl}}^{2} \sim M_{*}^{2+n} R^{n}$, where $M_{*}$ refers to the fundamental scale set by the higher-dimensional gravity. The hierarchy between $M_{\mathrm{Pl}}$ and $M_{*}$ can be very large if $R M_{*} \gg 1$. The ADD scenario is extremely attractive as not only the hierarchy problem is explained but also rich phenomenology of collider physics 困, 5, 6] and cosmology [7] are anticipated. In the near future, we may even be able to probe strong quantum gravity effects. On the other hand, as it stand now, the ADD scenario is afflicted by various unsolved fundamental problems. One of them is stabilization problem of the large extra dimensions or, equivalently, dynamical determination of large extra dimensionst. Paraphrasing, the central question posed by the ADD scenario is "Why are the extra dimensions so large?".

The second direction, as proposed by Randall and Sundrum (RS), is a scheme in which a five-dimensional gravity contains strongly gravitating three-branes, which induces a warped or nonfactorizable spacetime geometry. In the first RS model [9], which consists of two threebranes, a large mass hierarchy was achieved without large extra dimensions. In the second RS model [10], which consists only one three-brane of a positive tension, the extra dimension is noncompact yet the low-energy gravity on the three-brane still comes out to be effectively four-dimensional at low-energy, up to power-like corrections at short distance. This has to do with the fact that the five-dimensional graviton is decomposed into a massless bound state confined to the three-brane as well as a continuum of the Kaluza-Klein (KK) states. For low-energy processes on the three-brane, the bound state dominates over the KK states and gives rise to an inverse-square law provided the five-dimensional bulk curvature is sufficiently large. The RS scenario has been extended to various directions. One extension involves turning on a cosmological constant on the brane [11, 12]. Other extensions include higher dimensional embedding [13] and realizations of domain walls in gravity coupled to scalars [15, 16, 28, 14, 20]. Whether the RS scenario can be embedded into a more complete setting has been addressed within the setup of supergravity [17, 18] and string theories [19].

\footnotetext{
${ }^{2}$ For two extra dimensions, an interesting proposal to this problem has been put forward in [8]
} 
In Refs. 22, 23, 24, 25, 26, 27], attempts were made to embed these two scenarios by utilizing gravitating defects in higher dimensions. Cohen and Kaplan have considered a brane world, which may be viewed as a global string in two extra dimensions without bulk cosmological constant 22]. Its spacetime geometry is strongly warped and, similar to the case in $(2+1)$ dimensions, exhibits a curvature singularity at a finite distance from the string core. They argued that, imposing a unitary boundary condition, the location of the singularity may play a role of the boundary of the extra dimensions. Gregory claimed [23] that, in the presence of a negative bulk cosmological constant, there does not exist a non-singular global vortex solution which asymptotes to anti-de Sitter spacetime, and that a genuine non-singular global vortex can form a (physical) singularity-free warped geometry provided the extra-dimensions asymptote to a cylinder. Extensions to higher extra-dimensional defects (for example, a global monopole in three extra-dimensions) and to inclusion of gauge fields were discussed in Ref. [24, 26] and in Ref.[25, 26], respectively.

In this paper, we would like to present a new sort of brane-world scenario, which accommodates features of the above two scenarios and, most significantly, offers a topological mechanism of stabilizing a large mass hierarchy. Our setup will be, in a higher-dimensional anti-de Sitter spacetime background, a class of extremal black brane made of $p$-dimensional global defect, which shall be referred in what follows as "global black $p$-brane". We focus on the interior region inside the black $p$-brane horizon and study effective four-dimensional gravity at low energy on a 'Standard Model'-brane, which could be provided by the core brane or by a 'probe' brane located near the core of the interior region. A crucial feature of the interior region is that, even though asymptoting to an infinitely long throat, its volume is finite. As such, the 'Standard Model'-brane will behave locally as a domain wall embedded in an anti-de Sitter space. The bulk graviton in this background yields a massless localized mode on the $p$-brane, reproducing the correct $(p+1)$-dimensional gravity at long distance, in the same way as in the RS scenario. In our new-brane world scenario, a noteworthy feature is that the $p$-brane horizon size is interpretable as a compactification size, as the Planck scale $M_{\mathrm{Pl}}$ is determined in units of the fundamental scale $M_{*}$ and the horizon size $r_{\mathrm{H}}$ via:

$$
M_{\mathrm{Pl}}^{p-1} \sim M_{*}^{p-1+n} r_{\mathrm{H}}^{n}
$$

It also shows that, by varying the horizon size $r_{\mathrm{H}}$, the effective gravity on the 'Standard Model'brane interpolates between the RS scenario and the ADD scenario limits when more than one extra dimension are present. A large mass hierarchy is then simply translated into large horizon size. The most interesting feature of our scenario is that the horizon size $r_{\mathrm{H}}$, measured in units of the fundamental scale $M_{*}$, is determined by the topological charge $Q$ of the global $p$-brane. 
For instance, for $n=2$ and $p=3$,

$$
r_{\mathrm{H}}^{2}=\frac{16 \pi v^{2}}{M_{*}^{4}|\Lambda|} Q^{2} \quad \rightarrow \quad r_{\mathrm{H}} \sim \frac{1}{M_{*}}|Q| .
$$

A large mass hierarchy then follows by taking $|Q| \gg 1$. As a consequence, in our new braneworld scenario, the macroscopic compactification size is derived explicitly from a microscopic theory equipped with a fundamental scale and stabilization of the large hierarchy is ensured by the discrete and conserved nature of the $p$-brane topological charge $Q$.

\section{Global Black p-Brane Solutions}

Our starting point is a $D$-dimensional Einstein gravity coupled to a field theory of an $n$ component scalar $\boldsymbol{\Phi}^{a}(a=1, \cdots, n)$ ( $n$-vector model). We assume that the $O(n)$ global symmetry is spontaneously broken to $O(n-1)$ and, at the ground-state $\left\langle\Phi^{2}\right\rangle=v^{2}$, the vacuum energy density is negative. Dynamics of the theory is governed by the following action:

$$
S=\int d x^{D} \sqrt{g_{D}}\left[-\frac{M_{*}^{D-2}}{16 \pi}(R+2 \Lambda)-\frac{1}{2} g^{A B} \nabla_{A} \boldsymbol{\Phi} \cdot \nabla_{B} \boldsymbol{\Phi}-\frac{\lambda}{4}\left(\boldsymbol{\Phi}^{2}-v^{2}\right)^{2}\right],
$$

where $A, B, \cdots$ denote $D$-dimensional spacetime indices and $M_{*}$ sets a 'fundamental mass scale' of the theory. This theory admits $p$-dimensional topological solitons, where $(p+1)=D-n$. We shall refer as 'global $p$-brane' in what follows. Note that $\boldsymbol{\Phi}^{a}$ and $v$ have a mass dimension of $(p+n-1) / 2$ and the negative bulk cosmological constant $(\Lambda<0)$ has a mass dimension of 2 . We shall use the notation $\left\{x^{\mu}\right\}$ with $\mu=0, \ldots, p$ for the coordinates on the $p$-brane worldvolume, and $\left\{\mathbf{y}^{a}\right\}$ with $a=1, \ldots, n$ for the coordinates in the extra dimensions and are related to the spherical coordinates of $\mathbf{S}_{n-1}$ by the usual relations: $y^{a}=$ $\left(r \cos \theta_{1}, \cdots \cdots, r \sin \theta_{1} \cdots \cos \theta_{d-1}, r \sin \theta_{1} \cdots \sin \theta_{d-1}\right)$ with $r^{2}=\mathbf{y} \cdot \mathbf{y}$.

The global $p$-brane is defined by a topologically nontrivial mapping of the vacuum manifold $O(n) / O(n-1)$ to the extra dimensions $\mathbf{S}_{n-1}$. For a unit topological charge, we take ansätz for the scalar field configuration as:

$$
\boldsymbol{\Phi}^{a}=v f(r) \frac{\mathbf{y}^{a}}{r} .
$$

Here, for regularity of the $p$-brane, the spherically symmetric function $f(r)$ is required to satisfy $f(r)=0$ at the center and approach $f(r)=1$ outside core. For simplicity, in this paper, we shall take a version of 'thin-wall approximation' so that only the region outside the core 3 is considered. We take the following metric ansätz of Schwarzschild-type:

$$
d s^{2}=e^{2 N(r)} B(r) \hat{g}_{\mu \nu}(x) d x^{\mu} d x^{\nu}+\frac{d^{2} r}{B(r)}+r^{2} d \Omega_{n-1}^{2},
$$

\footnotetext{
${ }^{3}$ Detailed analysis without assumption $f(r)=1$ will be treated in Ref. 31].
} 
where $\hat{g}_{\mu \nu}(x)$ denotes the metric of $(p+1)$-dimensional subspace longitudinal to the global $p$-brane worldvolume.

With the ansätz Eqs.(2) and (3), one obtains, from the action Eq.(1), the following set of field equations:

$$
\begin{array}{r}
(p+1) B N^{\prime 2}+\frac{2 p+3}{2} B^{\prime} N^{\prime}+\frac{p}{4} \frac{B^{\prime 2}}{B}+B N^{\prime \prime}+\frac{1}{2} B^{\prime \prime}+\frac{n-1}{r}\left(B N^{\prime}+\frac{1}{2} B^{\prime}\right) \\
\quad+\frac{1}{p+1} \frac{\hat{R}}{e^{2 A} B}=-\frac{2}{p+n-1} \Lambda, \\
(p+1)\left(B N^{\prime 2}+\frac{3}{2} B^{\prime} N^{\prime}+B N^{\prime \prime}+\frac{1}{2} B^{\prime \prime}\right)+\frac{n-1}{2} \frac{B^{\prime}}{r}=-\frac{2}{p+n-1} \Lambda, \\
(p+1) \frac{B N^{\prime}}{r}+\frac{p+2}{2} \frac{B^{\prime}}{r}+(n-2) \frac{B-1}{r^{2}}=-\frac{8 \pi G_{D}}{r^{2}}-\frac{2}{p+n-1} \Lambda,
\end{array}
$$

where we have rescaled both the coordinates and the cosmological constant as $x_{A}\left(\equiv \sqrt{\lambda} v x_{A}\right)$, $\Lambda\left(\equiv \Lambda / \lambda v^{2}\right)$, respectively. We have also defined $G_{D} \equiv v^{2} / M_{*}^{D-2}$ and $\hat{R}$ for the curvature associated with the metric $\hat{g}_{\mu \nu}$.

The field equations Eqs.(4-6) being highly nonlinear, the spacetime around the global $p$ brane may develop singularities. As discussed in Ref. [24], global structure of the spacetime depends largely on the number of extra dimensions $n$, the bulk cosmological constant $\Lambda$, and the $p$-brane worldvolume curvature $\hat{R}$. If $\Lambda=0$, for $n=2$, the spacetime has a singularity, much as in the cosmic string background in four dimensions [22], while, for $n \geq 3$, the spacetime is regular and corresponds to that of the global monopole. In case $\Lambda<0$, the cosmological constant acts as a censor against the singularity, depending on the value of the worldvolume curvature $\hat{R}$. If $\hat{R}=0$, the spacetime is always regular no matter how small the cosmological constant may be. If $\hat{R} \neq 0$, the spacetime may become singular at a finite proper distance from the global $p$-brane core. For the black brane solutions, the case with $\hat{R}=0$ corresponds to an extremal black $p$-brane, while the case with $\hat{R}<0$ to a non-extremal black $p$-brane. What we expect is that, as in the case of the supergravity black $p$-brane, a singularity may be developed at the inner horizon of a non-extremal global $p$-brane [31]. For the most part of this paper, we will take that the $p$-brane is extremal and hence has a Ricci flat worldvolume, viz. $\hat{R}=0$.

Finding an exact analytic solution to the field equations Eq.(4)-(6) being impossible, let us try to examine behavior of the global $p$-brane at various regions. Outside the core, the spacetime is determined solely by the relative magnitude of the scalar field energy density $\left(8 \pi G_{D} / r^{2}\right)$ to the cosmological constant $(|\Lambda|)$. Analysis via series expansion and numerical integration indicates that the product $e^{2 N} B$, which determines the (00)-th component of the spacetime metric, decreases monotonically near the core for $2 \pi G_{D}>|\Lambda|$, and can be made to vanish at a finite distance by tuning $G_{D}$.

In the region far from the center where $8 \pi G_{D} / r^{2} \ll|\Lambda|$, the aforementioned metric functions 
behave as: $N(r) \sim N_{\infty}$ and $B(r) \sim B_{\infty} r^{2}$, where $N_{\infty}$ is a calculable constant (determined by matching the function to the interior-region) and $B_{\infty} \equiv 2|\Lambda| /(p+n)(p+n-1)$. We thus find that the exterior-region asymptotes to a $D$-dimensional anti-de Sitter spacetime $\left(A d S_{D}\right)$. By introducing a proper radial distance $\chi \equiv \int d r / \sqrt{B(r)}$, the spacetime in the exterior-region can be expressed as follows:

$$
d s^{2}:=e^{2 \sqrt{B_{\infty}} \chi} \bar{g}_{\mu \nu} d x^{\mu} d x^{\nu}+d \chi^{2}+e^{2 \sqrt{B_{\infty}} \chi} d \Omega_{n-1}^{2} .
$$

Here, $\bar{g}_{\mu \nu}(x)$ is a general Ricci-flat metric on the brane, satisfying $(p+1)$-dimensional vacuum Einstein field equation: $\bar{R}_{\mu \nu}(\bar{g})=0$.

The global $p$-brane horizon is where the timelike Killing vector $\partial_{t}$ becomes null and is formed in a region where the energy density of the scalar field and the bulk cosmological constant are comparable. Thus, we will take that there exists a horizon at a finite coordinate distance $r=r_{\mathrm{H}}$ from the $p$-brane center, viz., $\exp \left[2 N\left(r_{\mathrm{H}}\right)\right] B\left(r_{\mathrm{H}}\right)=0$. We will also assume that $B(r)$ vanishes at $r=r_{\mathrm{H}}$ and is analytic around $r=r_{\mathrm{H}}$. However, we will not impose further constraints on the function $\exp [2 N(r)]$, as it could become singular at $r_{\mathrm{H}}$, as is easily observed from Eq.(6).

Multiplying $e^{2 N} B$ to Eq. (四) and taking the limit $r \rightarrow r_{\mathrm{H}}$, we find $B^{\prime}\left(r_{\mathrm{H}}\right)=0$. As $B\left(r_{\mathrm{H}}\right)=$ $B^{\prime}\left(r_{\mathrm{H}}\right)=0$, at $r=r_{\mathrm{H}}$, the metric function $B(r)$ can be expanded as:

$$
B(r):=B_{\mathrm{H}}\left(r_{\mathrm{H}}-r\right)^{2}+B_{3}\left(r_{\mathrm{H}}-r\right)^{3}+\cdots .
$$

Inserting the expansion into Eq.(6), one finds

$$
N^{\prime}(r):=\frac{\beta}{\left(r_{\mathrm{H}}-r\right)^{2}}+\frac{\alpha}{\left(r_{\mathrm{H}}-r\right)}+\mathcal{O}\left[\left(r_{\mathrm{H}}-r\right)^{0}\right],
$$

where the coefficients $\alpha, \beta$ are given by:

$$
\begin{aligned}
\alpha & \equiv \frac{p+2}{p+1}\left[1-\frac{4|\Lambda|}{(p+2)(p+n-1) B_{\mathrm{H}}}\right] \\
\beta & \equiv \frac{-1}{p+1}\left[\frac{8 \pi G_{D}-n+2}{r_{\mathrm{H}}^{2}}-\frac{2|\Lambda|}{p+n-1}\right] .
\end{aligned}
$$

Apparently, Eq.(9) indicates that, as $r \rightarrow r_{\mathrm{H}}, N^{\prime}(r)$ is divergent quadratically and hence $e^{2 N} B$ is divergent exponentially. However, Eq.(4) forces the coefficient $\beta$ to be zero, as can be seen straightforwardly from substituting Eqs.(8) and (9) into Eq.(四). Thus, $N(r)$ is at most logarithmically divergent at $r=r_{\mathrm{H}}$. As $\beta=0$, from Eq.(11), we also obtain:

$$
r_{\mathrm{H}}^{2}=\frac{(p+n-1)\left(8 \pi G_{D}-n+2\right)}{2|\Lambda|} .
$$

This is one of the central relations we shall be using later for stabilizing large mass hierarchy. Using the series expansions given by Eqs.(8) and (9), we obtain from Eqs.(4) and (5) that:

$$
B_{\mathrm{H}}(1-\alpha)^{2}=\frac{2|\Lambda|}{(p+1)(p+n-1)},
$$


and, from simultaneous solution of Eqs.(12) and (10),

$$
(1-\alpha)=\frac{1}{4}[1+\sqrt{(p+9) /(p+1)}] .
$$

We have dropped the negative root of $(1-\alpha)$, as, in that case, the norm of $\partial_{t}$ will diverge at $r=r_{\mathrm{H}}$, leading to a contradiction with our starting assumption that $r_{\mathrm{H}}$ is the $p$-brane horizon. Then, near the horizon, the spacetime metric Eq.(3) has the form:

$$
d s^{2}:=B_{\mathrm{H}}\left[\sigma\left(r-r_{\mathrm{H}}\right)\right]^{2(1-\alpha)} \bar{g}_{\mu \nu} d x^{\mu} d x^{\nu}+\frac{d r^{2}}{B_{\mathrm{H}}\left(r-r_{\mathrm{H}}\right)^{2}}+r_{\mathrm{H}}^{2} d \Omega_{n-1}^{2} .
$$

Here, $\sigma$ is a signature factor taking value -1 in the interior region $\left(r<r_{\mathrm{H}}\right)$ and 1 in the exterior region $\left(r>r_{\mathrm{H}}\right)$ ft. It is easy to confirm that $r=r_{\mathrm{H}}$ is a degenerate horizon of the timelike Killing vector $\partial_{t}$ : $\partial_{t}$ becomes null at $r_{\mathrm{H}}$ and the surface gravity is zero. The surface at $r=r_{\mathrm{H}}$ is also the Cauchy horizon of a $(p+2)$-dimensional anti-de Sitter space. Hence, the spacetime Eq.(13) is simply the near horizon geometry of an extreme black $p$-brane, which is typically of the form $A d S_{p+2} \times \mathbf{S}_{n-1}$. The curvature radii of $A d S_{p+2}$ and $\mathbf{S}_{n-1}$ are given by $k^{-1} \equiv 1 / \sqrt{(1-\alpha)^{2} B_{\mathrm{H}}}$ and $r_{\mathrm{H}}$, respectively.

To see the spacetime structure, we find it convenient to reexpress Eq.(13) in the Poincaré coordinates by changing the radial variable as $\exp (-k \chi) \equiv \sqrt{B_{H}}\left[\sigma\left(r-r_{\mathrm{H}}\right)\right]^{1-\alpha}$ :

$$
d s^{2}:=\exp (-2 k \chi) \bar{g}_{\mu \nu} d x^{\mu} d x^{\nu}+d \chi^{2}+r_{\mathrm{H}}^{2} d \Omega_{n-1}^{2},
$$

where the curvature scale $k$ of the $A d S_{p+2}$ space is defined by $k \equiv(1-\alpha) \sqrt{B_{\mathrm{H}}} \sim \sqrt{|\Lambda|}$. For the interior metric $(\sigma=-1), \chi$ runs from a finite value to $\infty$ (at $\left.r=r_{\mathrm{H}}\right)$. For the exterior solution $(\sigma=1), \chi$ runs between $\infty\left(\right.$ at $\left.r=r_{\mathrm{H}}\right)$ and $-\infty($ at $r=\infty)$, but $\chi$ ought to be truncated at a finite distance as, at sufficiently large $r$, the near-horizon geometry is replaced by an asymptotic spacetime Eq.(17). The near-horizon geometry Eq.(14) coincides with the cigar-like spacetime geometry with an exponentially damping warp-factor discovered by [23] and [24]. Hence, among the solutions found in [23] and [24], the ones relevant for the RS scenario would be interpreted quite naturally as the near-horizon geometry of global black p-branes. Moreover, we also find that the two solutions, Eqs.(17) and (13), which were apparently treated disjointly in [23] and [24], can be matched to each other.

On the other hand, in the region between the $p$-brane core and the horizon, where the cosmological constant is negligible compared to the field energy density ? , the spacetime geometry depends on the transverse space dimensions. When $n=2$, the geometry of this region resembles that of the Cohen-Kaplan solution of Ref. [22]. If $n \geq 3$, the geometry of this region would become similar to that of the global monopole solution [24.

\footnotetext{
${ }^{4}$ Note that, so far, we have examined the near-horizon region from the inside toward $r_{\mathrm{H}}$ and hence obtained only the interior metric $(\sigma=-1)$. Had we done the analysis from the outside region, we would then obtain the exterior metric $(\sigma=1)$.

${ }^{5}$ If $\Lambda$ is much smaller than the fundamental scale $M_{*}^{2}$, this region overwhelms the near-horizon region.
} 
While we have not resolved yet whether the geometries obtained in separated regions can be connected mutually or not, painstaking numerical calculations indicate that the black $p$-brane solution does exist. Putting the above results together, the spacetime geometry of the global p-brane is illustrated in Figure 1.

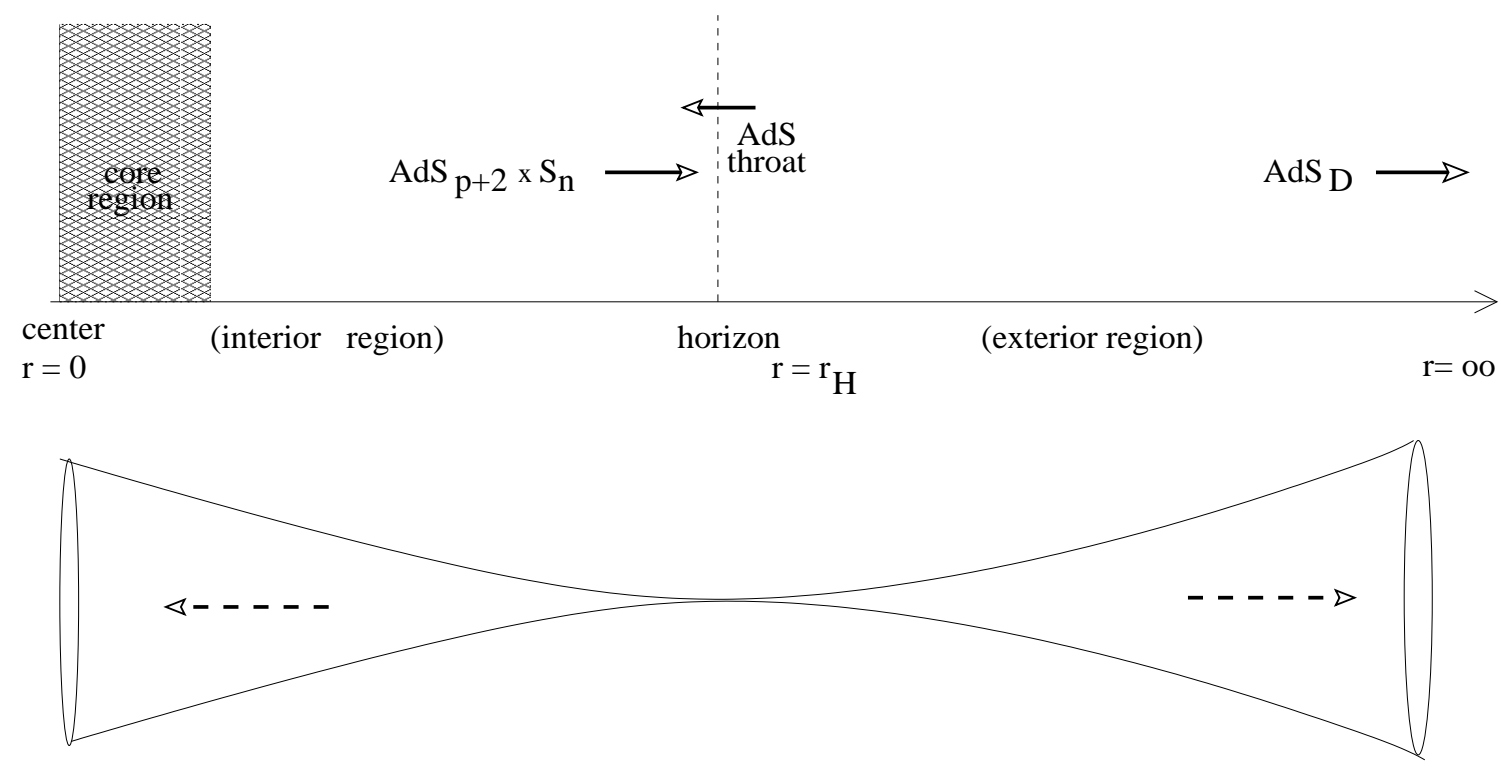

Figure 1: (a) Spacetime geometry of the global p-brane, (b) Corresponding warped geometry in Poincaré coordinate.

\section{Effective Theory of The New Brane-World Gravity}

Having understood the spacetime geometry generated by the global $p$-brane, we now proceed to a new brane-world scenario. The first step would be identifying a suitable slice of the spacetime geometry, where an effective $(p+1)$-dimensional gravity may arise at low-energy.

The 'exterior region' by itself does not seem to provide a suitable framework for the braneworld scenario, as, in one direction, one will reach the $(p+2)$-dimensional AdS horizon while, in the other direction, one will reach the $D$-dimensional AdS spacetime. On the other hand, the interior region possesses all the features necessary for realizing the $\mathrm{RS}$ scenario. In one direction, it asymptotes to the AdS throat $\left(A d S_{p+2} \times \mathbf{S}_{n-1}\right)$. As such, the central region surrounded by the AdS region looks like a one-sided RS domain-wall embedded in $(p+2)$-dimensional AdS spacetime, viz. a smooth Planck-brane in the RS scenario.

In the RS scenario, as is investigated extensively, the standard $(p+1)$-dimensional Newtonian gravity is reproduced in the brane-world provided a massless $(p+1)$-dimensional graviton is localized on the $p$-brane. In the new brane-world scenario, existence of the massless graviton 
is evident, as the field equations Eqs.(4)-(6) permits always a solution with a general Ricci flat metric $\bar{g}_{\mu \nu}(x)$ - the massless graviton is simply the standard travelling gravitational wave of the linearized $(p+1)$-dimensional vacuum Einstein's field equation. Then, the massless $(p+1)$ dimensional graviton is localized on the $p$-brane provided the $(p+1)$-dimensional Planck scale $M_{\mathrm{Pl}}$ is finite. From the $(p+1)$-dimensional effective action, one identifies the Planck scale with:

$$
M_{\mathrm{Pl}}^{p-1}=M_{*}^{D-2} \int d y^{n} \sqrt{g_{D}} g^{00} \sim M_{*}^{p-1+n} r_{\mathrm{H}}^{n} .
$$

The relation Eq.(15) shows that the $(p+1)$-dimensional Planck scale is determined by the fundamental scale $M_{*}$ and the horizon size $r_{\mathrm{H}}$ quite analogous to the usual Kaluza-Klein theories except that now the size of the compact extra dimension in the Kaluza-Klein theories is played by the size of the horizon $r_{\mathrm{H}}$. Hence, in the new brane-world scenario, we propose to interpret the horizon size $r_{\mathrm{H}}$ as an effective size of the noncompact $n$-dimensional space, even though the interior region extends to infinity. The $n$ extra dimensions turn into a direct product of an $(n-1)$-dimensional sphere $\mathbf{S}_{n-1}$ of a fixed radius $r_{\mathrm{H}}$ times an infinitely extended radial direction. Despite being noncompact, due to the warped spacetime geometry, the radial direction yields a finite effective size $\sim r_{\mathrm{H}}$.

Indeed, to an observer living outside the horizon (the exterior region), the above interpretation seems correct. The interior region occupies only a finite part of the higher dimensional transverse space of volume $\sim r_{\mathrm{H}}^{n}$ and the apparent infinite extension of the interior region is simply a result of the warping of the extra dimensions by the gravity produced by the $p$-brane itself. Later, we will support the interpretation further with analysis of the effective $(p+1)$ dimensional gravity.

To see the behavior of $(p+1)$-dimensional gravity on the global $p$-brane, let us study small perturbations of the spacetime metric: replacing $\bar{g}_{\mu \nu}(x)$ with $\eta_{\mu \nu}+h_{\mu \nu}(x, z)$ in Eq.(3). Imposing the so-called Randall-Sundrum gauge, one can easily find linearized field equation for $h_{\mu \nu}$. For a general metric of the form in Eq.(3), after a change of variables $\xi=\int^{r} \sqrt{-g^{00}\left(r^{\prime}\right) g_{r r}\left(r^{\prime}\right)} d r^{\prime}$, $\widetilde{h_{\mu \nu}}=K h_{\mu \nu}$ and a separation of variables $\widetilde{h_{\mu \nu}}(\xi, \Omega)=\epsilon_{\mu \nu} e^{i p \cdot x} \mathcal{R}_{m \ell}(\xi) Y_{\ell}(\Omega)$, where $\epsilon_{\mu \nu}$ is the polarization tensor and $Y_{\ell}(\Omega)$ is the $n$-dimensional spherical harmonics, it can be shown that the linearized field equation can always be expressed as an 'analog' non-relativistic Schrödinger equation:

$$
\left[-\frac{\partial^{2}}{\partial \xi^{2}}+V_{\text {eff }}(\xi)\right] \mathcal{R}_{m \ell}(\xi)=m^{2} \mathcal{R}_{m \ell}(\xi)
$$

in which

$$
V_{\mathrm{eff}}(\xi) \equiv \frac{K^{\prime \prime}(\xi)}{K(\xi)}+\ell(\ell+n-2) \frac{g_{00}(\xi)}{r(\xi)^{2}} .
$$

Here, $K \equiv r^{(n-1) / 2} g_{00}^{p / 4}$ and $m(=\sqrt{-p \cdot p})$ denotes the $(p+1)$-dimensional Kaluza-Klein mass of the continuum modes. The zero-mode wave function, with $m=\ell=0$, is easily identified 
with $R_{00}(\xi)=K(\xi)$. All the low-energy physics in the brane-world can then be analyzed qualitatively from the structure of the effective potential $V_{\text {eff }}(\xi)$.

Consider a point particle of mass $m^{*}$ localized on the global $p$-brane. Then, the Newtonian potential is calculated straightforwardly as:

$$
U(|\mathbf{x}|)=-G_{p+1} \frac{m^{*}}{|\mathbf{x}|^{p-3}}-\frac{m^{*}}{M_{*}^{n+2}} \sum_{\ell} \int_{m \neq 0} d m \rho(m)\left|\mathcal{R}_{m \ell}(0)\right|^{2} \frac{e^{-m|\mathbf{x}|}}{|\mathbf{x}|^{p-3}} .
$$

Here, $\rho(m)=m^{\delta}$ denotes the density of states for the continuum modes. If the extra dimensions are noncompact, it follows that $\delta=n-1$, as the $n$-dimensional plane-waves yields a density of states $m^{n-1}$ (up to a calculable numerical coefficient). In the present case, $(n-1)$-extra dimensions are compactified to a sphere $\mathbf{S}_{n-1}$ of radius $r_{\mathrm{H}}$. Thus, the lower partial-wave modes $(\ell \sim 1)$ would behave as plane waves only in the radial direction, while higher partial-wave modes $(\ell \gg 1)$ would behave as plane waves in the entire extra dimensions. This implies that $\delta$ will depend on $\ell$ and interpolates $\delta=0$ at small $\ell$ and $\delta=n-1$ at large $\ell$.

The right-hand side of Eq.(11) ought to be positive. This condition yields $v^{2}>(n-$ $2) / 8 \pi M_{*}^{D-2}$. While the condition does not give any new information for $n=2$, for $n>2$, the condition sets the symmetry breaking scale $v$ to be of the oder of the fundamental scale $M_{*}$. On the other hand, in order to trust the global $p$-brane profile, $v^{2 / D-2}$ ought to be smaller than the fundamental scale: $v^{2 / D-2}<M_{*}$. Hence, we will be assuming that $(n-2) / 8 \pi<v^{2} / M_{*}^{D-2}<1$. We will also assume that $v^{2 / D-2} \sim M_{*}$ for $n=2$, but this seems valid for naturalness reasons. The horizon size $r_{\mathrm{H}}$ and the anti-de Sitter curvature radius $k$ are then determined by the bulk cosmological constant $\Lambda$, viz, $k^{2} \sim r_{\mathrm{H}}^{-2} \sim|\Lambda|$. In models with RS-type scenario, the bulk cosmological constant $\Lambda$ had always been identified with the fundamental scale. However,

given our ignorance concerning the cosmological constant problem, there is a priori no reason to expect that $\Lambda$ is of the same order as the fundamental scale $M_{*}^{2}$. Thus, we shall be treating $\Lambda$ simply as an input parameter. The only experimental input is that, from the present-day gravity measurement, $r_{\mathrm{H}}$ ought to be smaller than $\sim 1 \mathrm{~mm}$ if it is to be interpreted as a compactification scale. In what follows, we will consider two limiting values of $\Lambda:|\Lambda| \sim M_{*}^{2} \sim M_{\mathrm{Pl}}^{2}$ and $|\Lambda| \sim 10^{6-60 / n} \mathrm{GeV}^{2} \ll M_{*}^{2} \sim m_{\mathrm{EW}}^{2}$ for $p=3$. We will call the first limit as Randall-Sundrum (RS) limit and the second as large extra dimension (ADD) limit and explore physics in each of them in detail.

\subsection{Randall-Sundrum Limit}

In the RS limit, the interior region is well approximated by the near-horizon geometry Eq.(14), as both the core radius $r_{c} \sim(\sqrt{\lambda} v)^{-1 / 2}$ and the horizon size $r_{\mathrm{H}}$ are of the order of the fundamental scale. As such, $8 \pi G_{D} / r^{2}$ and $|\Lambda|$ are of comparable magnitude over the entire 'interior region'. The curvature radii of $A d S_{p+2}$ and $\mathbf{S}_{n-1}$ are of the order of the Planck scale. Thus, at 
low-energy below the Planck scale, the extra space is reduced effectively to a one-dimensional space. Consequently, the global $p$-brane core looks like a $p$-dimensional domain-wall embedded in an $A d S_{p+2}$ bulk spacetime. In the 'thin-wall approximation' limit, the situation is essentially the same as that of the RS scenario. After a change of variable $k \xi+1=\exp (k \chi)$, one obtains the effective potential $V_{\text {eff }}(\xi)$ as

$$
V_{\mathrm{eff}}(\xi)=\left(\frac{p(p+2) k^{2}}{4}+\frac{\ell(\ell+n-2)}{r_{\mathrm{H}}^{2}}\right) \frac{1}{(k \xi+1)^{2}} .
$$

The 'analog' Schrödinger equation Eq.(16) allows a normalized zero-mode $\mathcal{R}_{00} \sim r_{\mathrm{H}}^{-n / 2}(k \xi+$ $1)^{-p / 2}$ and a continuum of Kaluza-Klein modes given by a linear combination of Bessel functions $\mathcal{R}_{m \ell}(\xi)=(\xi+1 / k)^{1 / 2}\left[a_{m \ell} Y_{\nu}(m(\xi+1 / k))+b_{m \ell} J_{\nu}(m(\xi+1 / k))\right]$, where $a_{m \ell}$ and $b_{m \ell}$ are $m$ dependent coefficients, and $\nu \equiv \frac{p+1}{2} \sqrt{1+4 \ell(\ell+n-2) /(p+1)^{2} k^{2} r_{\mathrm{H}}^{2}}$. As anticipated, for $\ell=$ 0 partial wave, the potential and the wavefunction are precisely the same as those of the original RS model. A difference from the RS model arises for massive Kaluza-Klein modes with $\ell \neq 0$ extended in $A d S_{p+2}$ - they originate from the Kaluza-Klein reduction of the fundamental $D$-dimensional bulk fields on $\mathbf{S}_{n-1}$. Consequently, they exhibit a discrete mass spectrum proportional to $\ell(\ell+n-2) / r_{\mathrm{H}}^{2}$.

In order to evaluate the sub-leading contributions due to the Kaluza-Klein modes to the $(p+1)$-dimensional static gravitational potential, one will need to estimate $\mathcal{R}_{m \ell}(\xi=0)$ for $m \ll M_{*}$. As the $p$-brane core region is extended over the scale $r_{c}$ in the transverse space, it is difficult to impose a suitable boundary condition, in contrast to the original RS model. However, in case the central region of the Schrödinger potential is localized within the AdS scale $1 / k$, the sub-leading contribution would be identical to those of the original RS limit (The same argument has been put forward earlier by [28].).

Adopting the same procedure as in [28], we obtain the value of the radial wavefunction on the brane at $\xi=0$ at leading order in small $m$ expansion as $\mathcal{R}_{m \ell}(\xi \simeq 0) \sim(m / k)^{\nu-3 / 2}$ for $m \ll k$. It is now straightforward to calculate the static gravitational potential at long distances $|\mathbf{x}| \gg 1 / M_{\mathrm{Pl}}$ generated by a point particle of mass $m^{*}$ located on the $p$-brane:

$$
U(|\mathbf{x}|) \sim-G_{p+1} \frac{m^{*}}{|\mathbf{x}|^{p-2}}\left[1+\left(\frac{1}{k|\mathbf{x}|}\right)^{p-1}+\sum_{\ell \neq 0}\left(\frac{1}{k|\mathbf{x}|}\right)^{\delta+2 \nu-2}\right] .
$$

The leading term originating from the massless bound-state graviton contribution is the usual $(p+1)$-dimensional Newtonian potential. The second correction term coming from continuum modes with $\ell=0$ is suppressed enormously for $k$ of order the $(p+1)$-dimensional Planck scale and the distance scale the gravity is tested. The correction grows like $1 / r^{p-1}$ rather than $1 / r^{n+1}$, unlike those expected for the RS-type models with $n$ extra dimensions [20, 26, 27]. The difference from the RS scenario arises only through the third term, which originates from the 
contribution of modes with $\ell \geq 1$ and was absent in the original RS model. The information of the extra $(n-1)$ dimensions is reflected through this term only. It, however, is more strongly suppressed than the second term for large $\ell$. Thus, the low-energy processes on the brane are indistinguishable from those in the original RS scenario.

\subsection{Large Extra-Dimension Limit}

Consider next the case where the cosmological constant $\Lambda$ is hierarchically smaller than the fundamental mass scale $M_{*}$. In this situation, the physics will be quite analogous to that of the ADD scenario [1]. Adopting the results of [1], in our new brane-world scenario, phenomenologically acceptable size of the horizon and the cosmological constant are $r_{\mathrm{H}} \sim 10^{30 / n-17} \mathrm{~cm}$ and $|\Lambda| \sim 10^{6-60 / n} \mathrm{GeV}^{2}$, respectively, with $M_{*} \sim m_{\mathrm{EW}} \sim 10^{3} \mathrm{GeV}$ and $M_{\mathrm{Pl}} \sim 10^{19} \mathrm{GeV}$ for $p=3$. In this case, in the transverse space, the core region and the $A d S_{p+2}$ throat region are squeezed into small portions and, in between the two, most portion is occupied by an intermediate region characterized by $8 \pi G_{D} / r^{2} \gg|\Lambda|$. The proper size of the intermediate region $\xi_{\mathrm{I}}$ is close to the horizon size: $\xi_{\mathrm{I}} \sim r_{\mathrm{H}}$. As such, depending on details of the geometry of this region, the functional form of the 'analog' Schrödinger potential will be modified from Eq.(19).

In fact, as mentioned earlier, the geometry of the intermediate region can be approximated by that of the Cohen-Kaplan solution [22] for $n=2$ and those of global monopoles [24] for $n \geq 3$. Let us analyze them in more detail. For $n=2$, the 'analog' Schrödinger potential is attractive near the core and shoots to a negative infinity at the singularity [22]. This potential ought to be excised at a point near the singularity and then glued smoothly to a potential generated by the $A d S_{p+2}$ throat region, Eq.(19). As the potential takes a negative value in this region, the continuum Kaluza-Klein modes are separated by a potential barrier whose peak is located at the $A d S_{p+2}$ throat region and height is given by $\sim k^{2}$. Furthermore, the potential extends over the $A d S_{p+2}$ curvature scale, $1 / k$. As discussed in the previous subsection, in case the Schrödinger potential is localized within the scale $1 / k$, the effect of light Kaluza-Klein continuum modes (whose mass is ranged $m \ll k$ ) turns out identical to that in the RS limit. Hence, the subleading contribution to the long-range static gravitational potential should be the same as in the RS limit, independent of details of the potential profile. As a result, we will obtain the static gravitational potential with corrections of the same form as Eq.(20) (at a long distance, $\left.|\mathbf{x}| \gg r_{\mathrm{H}} \sim k^{-1}\right)$. The only difference is that the horizon size $r_{\mathrm{H}}$ is now macroscopically large. The continuum Kaluza-Klein modes with masses $m \gg k$ would pass over the potential and hence are unsuppressed at the core: $\mathcal{R}_{m \ell}(0) \sim 1$. Hence, at a short distance $|\mathbf{x}| \ll r_{\mathrm{H}} \sim k^{-1}$, the static gravitational potential is just $(p+3)$-dimensional:

$$
U(|\mathbf{x}|) \sim \frac{1}{M_{*}^{p+1}} \frac{m^{*}}{|\mathbf{x}|^{p}}
$$


When $n \geq 3$, the intermediate region $\left(\xi \lesssim r_{\mathrm{H}}\right)$ is approximated by the global monopole geometry 24]:

$$
d s^{2}=\eta_{\mu \nu} d x^{\mu} d x^{\nu}+d \xi^{2}+\gamma^{2} \xi^{2} d \Omega_{n-1}^{2}
$$

where $\gamma^{2} \equiv 1-8 \pi G_{D} /(n-2)$ represents the solid angle deficit in extra dimensions. In this region, the 'analog' Schrödinger potential is repulsive of the form:

$$
V_{\mathrm{eff}}(\xi)=\left[\frac{(n-1)(n-3)}{4}+\frac{\ell(\ell+n-2)}{\gamma^{2}}\right] \frac{1}{\xi^{2}},
$$

The potential in the $A d S_{p+2}$ region can be obtained from replacing $\xi$ by $\xi-\xi_{I}$ in Eq.(19).

For $n=3$, Eq.(23) indicates that, in the intermediate region, the repulsive potential term vanishes identically and hence imposes no further suppression for the Kaluza-Klein continuum modes. As such, the physics on the $p$-brane would be the same as that of the ADD scenario with $n=3$. We thus find that, for $n=2,3$, the effective low-energy gravity behaves as expected in a world with $n$-extra compactified dimensions of radius $r_{\mathrm{H}}$.

For $n \geq 4$, the physics on the $p$-brane will be quite different from that of $n=2,3$, mainly due to the repulsive potential in the intermediate region. As $\xi_{\mathrm{I}} \sim 1 / k$, the potentials in the two regions are matched smoothly at $\xi_{\text {I }}$ and the potential increases monotonically up to $\sim M_{*}^{2}$ as approached to the core region $r_{c} \sim(\sqrt{\lambda} v)^{-1} \sim M_{*}^{-1}$. In this region, the Kaluza-Klein continuum modes are given by combinations of Bessel functions $\sqrt{\xi}\left[A_{m \ell} J_{v}(m \xi)+B_{m \ell} Y_{v}(m \xi)\right]$ where $v \equiv$ $\left(\frac{n-2}{2}\right)\left[1+4 \ell(\ell+n-2) /(n-2)^{2} \gamma^{2}\right]^{1 / 2}$. The modes with mass $m \lesssim M_{*}$ are suppressed at the core and, at leading order in $m$, is given by $\mathcal{R}_{m \ell}(0) \sim\left(m / M_{*}\right)^{v-3 / 2}$. As such, the static gravitational potential generated by a point particle with mass $m^{*}$ at distances $|\mathbf{x}| \gg M_{*}^{-1}$ located on the $p$-brane is simply obtained by replacing $k, \nu$ with $M_{*}, v$ in Eq.(20).

Actually, this regime is phenomenologically interesting as the physics on the $p$-brane is different from those of the RS- or the ADD-limits. For the RS scenario, by naturalness, the fundamental scale and the bulk cosmological constants are assumed to be of the same order as the Planck scale $M_{\mathrm{Pl}}$. Moreover, the gravity in the brane-world is not significantly modified over the 33 orders of magnitude between $\sim 1 \mathrm{~cm}$ and the Planck length $M_{\mathrm{Pl}}^{-1} \sim 10^{-33} \mathrm{~cm}$. For the ADD scenario, the fundamental scale may be taken of the order of the electro-weak scale $m_{\mathrm{EW}}$ but, to obtain the correct Planck scale, the compactification size ought to be macroscopically large, leading to very light continuum modes. Consequently, the gravity in the brane-world changes from four- to higher-dimensional gravity around the compactification scale, much larger than the electro-weak scale $1 / m_{\mathrm{EW}}$. However, in the last case with $n \geq 4$, the continuum modes lighter than the fundamental mass scale $\sim m_{\mathrm{EW}}$ are suppressed and the gravity in the braneworld remains $(p+1)$-dimensional down to the distance $\sim m_{\mathrm{EW}}^{-1}$. Nor do very light moduli fields associated with the large extra dimensions $\mathbf{S}_{n-1}$ get problematic, as they are strongly suppressed by the potential barrier of height $M_{*}^{2} \sim m_{\mathrm{EW}}^{2}$. 


\section{Large Mass Hierarchy from Large Horizon Size}

So far, we have obtained a new brane-world, whose spirit is similar to ADD scenario for $n=2,3$ but distinct for $n \geq 4$. In all cases, the large mass hierarchy is explained by a large horizon size $r_{\mathrm{H}}$. Recall that a large horizon size is provided by a small bulk cosmological constant. As such, the hierarchy problem is now transmuted into a large disparity between the fundamental scale and the small bulk cosmological constant. This hierarchy may be stable in that a small change of the bulk cosmological constant impart little influence on the low-energy dynamics on the brane-world as expected in the conventional large extra dimension scenario. Then, remaining question is whether the mass hierarchy can be set large by underlying dynamics. Generically, this is impossible as radiative corrections would drive the bulk cosmological constant to the value set by the fundamental mass scale, possibly except supersymmetric bulk theory.

We now point out a novel mechanism for maintaining a large mass hierarchy in the new brane-world without fine-tuning of various scales involved. We begin with an observation that, for the global $p$-branes, the fundamental scale can be separated from the horizon scale parametrically large. Recall that, for the extremal $p$-brane, the horizon size $r_{\mathrm{H}}$ is proportional to the global charge $Q$ carried by the brane. The only remaining question is whether the $p$-brane remains stable once its global charge $Q$ is cranked up to a large value. Note that, so far, we have restricted our considerations to the $|Q|=1$ case, as, for $n \geq 3$ (global (hyper)-monopoles), they are the only ones compatible with spherical symmetry and regularity at the origin. On the other hand, for $n=2$, a rotationally symmetric solution with an arbitrarily large $Q$ is possible!

The $n=2$ configuration with a large winding number $Q$ is obtained simply by replacing $8 \pi G_{D}$ in the unit charge configuration with $8 \pi G_{D} Q^{2}$. Then, from Eq.(11), we see that the horizon size is given, in terms of the unrescaled parameters, by

$$
r_{\mathrm{H}}^{2}=\frac{4 \pi v^{2}(p+1)}{M_{*}^{p+1}|\Lambda|} Q^{2},
$$

whereas the curvature of the $A d S_{p+2}$ is unchanged and is determined solely by the bulk cosmological constant. We further assume most conservatively that there exists only one physical scale, viz. all scales are set by the fundamental scale. In this case, the horizon size is simply estimated as:

$$
r_{\mathrm{H}} \sim \frac{1}{M_{*}}|Q|
$$

Putting $M_{*} \sim m_{\mathrm{EW}} \sim 10^{3} \mathrm{GeV}$ and that $r_{\mathrm{H}}$ and $|Q|$ are chosen to reproduce the observed 4D Planck scale $M_{\mathrm{Pl}} \sim 10^{19} \mathrm{GeV}$ yields, for $p=3$, we find $Q \sim 10^{16}$. Hence, an extremal 3-brane with large global charge, $Q \sim 10^{16}$, provides for a dynamical determination of the large hierarchy between the Planck scale and the electro-weak scale despite the microscopic theory 
contains only one physical scale. Moreover, the large compactification size is now stabilized via topological mechanism, as the global charge is discrete and conserved.

The only drawback with the above mechanism is that, the $p$-brane thickness $r_{c}$ may become much larger than $1 / m_{\mathrm{EW}}$, as the thickness is proportional to the global charge $Q$. This does not look like the world we live in. Nevertheless, we could avoid this drawback, provided the thickness $r_{c}$ is much smaller than the horizon size, viz. $m_{\mathrm{EW}}^{-1} \ll r_{c} \ll r_{\mathrm{H}}$. In this case, one obvious strategy is to place the 'Standard Model'-brane not at the center but somewhere between $r_{c}$ and $r_{\mathrm{H}}$, similar in spirit to Ref. [29, 20]. The 'Standard-Model' brane should be much thinner than the centrally located 3-brane of charge $Q$, viz. a 3-brane with a small topological charge.

There are two options of the 'Standard-Model'-brane location, $r_{\mathrm{SM}}$. One option is to put it in the $A d S_{5}$ region: $r_{\mathrm{SM}} \sim r_{\mathrm{H}}$, as in Ref. 29, 20]. In this case, as the graviton amplitude is exponentially suppressed, one needs to fine-tune the location of the 'Standard Model'-brane with high precision in order to obtain the correct ratio between the Planck and the electroweak scales. Another option is to put it somewhere between the core and the $A d S_{5}$. In this region, the graviton amplitude falls off slowly and no fine-tuning is required for the the location of the 'Standard Model'-brane. The physics on the 'Standard-Model' brane would be the same as in the conventional large extra dimension scenario. In the case of a large topological charge, the behavior of the continuum modes is more interesting. The horizon size is much larger than the fundamental length scale, while the hight of the 'analog' Schrödinger potential is $\sim m_{\mathrm{EW}}^{2}$. The central part of the potential then is much larger than the curvature length scale; $\xi_{\mathrm{I}} \sim r_{\mathrm{H}} \gg m_{\mathrm{EW}}^{-1}$. Thus, we cannot directly apply the procedure of Ref. 28], as we did in previous subsections to see the limiting behavior of the continuum modes in the central region of the analog Schrödinger potential. This regime seems to mimic the large box limit of Ref. 30, which consists of a slice of 5D flat Minkowski space glued between two 5D AdS regions. In that setup, when the size of the box is large compared to the anti-de Sitter scale, there are resonant modes with enhanced support inside the box. Even though there is a continuum of bulk modes with masses near the resonant mass, their net contribution mimics a single normalizable mode with this resonant mass. Thus the details of the anti-de Sitter region are suppressed in this setup. That is, the leading order corrections to the Newtonian potential are identical to those as if we were considering a compactification of flat five-dimensional spacetime on $\mathbf{S}^{1} / \mathbf{Z}_{2}$ orbifold. The regime with large winding number clearly resembles this large box limit of Ref. 30] and corresponds to the generalization of their setup [30] to a spacetime with more than five dimensions. The interior region has a finite portion of a space, even though it is not a flat space, surrounded by an anti-de Sitter region. Following the works of Ref. [30], we easily deduce that there ought to be resonant modes with masses $m_{\ell} \sim \ell / r_{\mathrm{H}}$, where $\ell=1,2,3, \ldots$. Hence, the low energy physics on the brane imitates the conventional large extra dimension 
scenario.

The phenomenology on the 'Standard Model' brane located in the region between the core and the anti-de Sitter region resembles closely that of the large extra dimension scenario except that the size of the large extra dimensions is provided by the large topological charge carried by the global black 3-brane and is stabilized via the topological charge conservation. Stated differently, a global black brane with a large topological charge can determine dynamically a stable hierarchy between the four-dimensional Planck scale and the electroweak scale in a higher-dimensional theory with a single fundamental scale.

\section{Thinning Out Cosmological Constant via Hawking Ra- diation}

Many interesting attempts for the resolution of the cosmological constant problem have been claimed within the brane world scenarios [21]: If our four-dimensional world is embedded in a higher dimensional spacetime, changes of the vacuum energy of the brane, the brane tension, may affect the curvature in the extra dimensions only, retaining a Poincaré invariant fourdimensional worldvolume. These scenarios have undesirable features, such as the presence of naked singularity parallel to the brane, or the necessity of very specific form of the couplings in the effective action of the theory.

In this section, we would like to propose one intriguing piece of physics related to the cosmological constant - relaxation of the cosmological constant of the brane world via Hawking radiation. Till now, we have considered only the extremal black brane, of which world volume is Ricci-flat. For such extremal black branes, the Hawking temperature is zero because the surface gravity vanishes at the horizon. Therefore, there would be no Hawking radiation either.

On the other hand, a non-extremal black brane has nonzero Hawking temperature and radiates, evolving toward an extremal black brane. We take this as a possible mechanism for relaxing the cosmological constant in the brane world scenario. In general, excitations of the extremal black $p$-brane would convert the metric $\eta_{\mu \nu}$ to be either non-Ricci flat $\hat{g}_{\mu \nu}=\hat{g}_{\mu \nu}(x)$ such that $\hat{R}(\hat{g}) \neq 0$, or even to depend on the extra dimension coordinates $\hat{g}_{\mu \nu}=\hat{g}_{\mu \nu}(x, y)$. The non-extremal black $p$-brane produced so will be equipped with nonzero Hawking temperature. Subsequently, the excitations will be diluted via Hawking radiation.

In this picture, the nonzero vacuum energy of the brane is translated into the constant worldvolume curvature $\hat{R}(\hat{g}) \neq 0$, which would render the initially extremal black $p$-brane into a non-extremal one. This observation implies that, even though the Poincaré invariance along the black $p$-brane worldvolume direction is broken due to the change of the vacuum energy density of the brane, e.g., the quantum corrections to the brane tension, it can be recovered through 
a dilution mechanism of the excitation via the Hawking radiation process. Consequently, one would expect that no cosmological constant on the brane would be generated, at least, at asymptotic future. In this picture, the observed smallness of the cosmological constant could be explained, provided that our world brane is embedded in the interior region of a very nearextremal black brane. Moreover, the fact that the entropy density of our Universe is extremely small (but nonzero) compared to the entropy density usually expected from black hole physics seems to support it 9 .

The above argument is applicable as well for other problems associated with the brane world scenario. First, the process provides a new solution to the cosmological flatness problem. Even though the $p$-brane worldvolume might be bent initially, the bending energy ought to be diluted as the black brane Hawking radiates. The $p$-brane would evolve again toward the extremal one at asymptotic future. Second, the new flatness problem[33] associated with the approximate Lorentz invariance in the $p$-brane worldvolume direction may also be explained, as the bulk curvature generated by $\hat{g}_{\mu \nu}(x, y)$ could be diluted away via the aforementioned Hawking radiation.

\section{Summary and Discussion}

In this paper, we have put forward a new kind of brane-world scenario. The scenario is based on the existence of a global black p-brane in a higher-dimensional gravity. It represents a black hole-like topological soliton and is a $p$-dimensional extended object surrounded by a degenerated horizon. The geometry is perfectly regular everywhere. We have found that the interior region of such a global black brane possesses all of the features necessary for realizing the RS-type brane-world scenario. In this picture, the size of the horizon can be interpreted as the effective size of $n$ compact extra dimensions in that the Planck scale $M_{\mathrm{Pl}}$ is determined by the fundamental scale $M_{*}$ and the horizon size $r_{\mathrm{H}}$ via the familiar relation $M_{\mathrm{Pl}}^{p-1} \sim M_{*}^{p-1+n} r_{\mathrm{H}}^{n}$.

If the fundamental scale and the bulk cosmological constant are taken to be the brane world Planck scale, i.e., $M_{*} \sim|\Lambda|^{1 / 2} \sim M_{\mathrm{Pl}}$, then the size of the horizon is of the order of the Planck scale, $r_{\mathrm{H}} \sim M_{\mathrm{Pl}}$, and the low energy physics is essentially the same as in the original $\mathrm{RS}$ scenario. That is, the corrections to the Newtonian gravity have precisely the same power law behavior as in the RS scenario, despite the existence of more than one extra dimensions.

In case $M_{*} \sim m_{\mathrm{EW}}$, the horizon size should be large to reproduce the correct brane world Planck scale. When $n=2,3$, the gravity behaves as expected in a world with $n$ extra dimensions compactified to a size $r_{\mathrm{H}}$. On the other hand, for $n \geq 4$, there are significant changes in the low-energy physics. In this case, the continuum modes with mass $m<m_{\mathrm{EW}}$ are suppressed

6 The present entropy density of our universe is $\sim 10^{3} / \mathrm{cm}^{3}$, while for a four-dimensional black hole which has a Schwarzschild radius of order $1 \mathrm{~cm}$, the entropy is $\sim 10^{66}$. 
and the gravity on the brane is maintained to be $(p+1)$-dimensional down to distances of $\sim m_{\mathrm{W}}^{-1}$, in contrast to the conventional large extra dimension scenarios. Moreover, there are no light moduli fields associated with the large size of $\mathbf{S}^{n-1}$ as they are strongly suppressed in the central region. The hierarchy between $M_{\mathrm{Pl}}$ and $m_{\mathrm{EW}}$ is supported by large size of the horizon. However, there still remains a hierarchy between the weak scale and the tiny bulk cosmological constant is needed for a large horizon size.

Finally, in case the theory has intrinsically only one physical scale of the weak scale, i.e., $M_{*} \sim|\Lambda|^{1 / 2} \sim m_{\mathrm{EW}}$, the large horizon size is provided by a large winding number, $r_{\mathrm{H}} \sim$ $|Q| m_{\mathrm{EW}}$, without introducing small cosmological constant. The large hierarchy between $M_{\mathrm{Pl}}$ and $m_{\mathrm{EW}}$ is now provided by the large winding number of the global black $p$-brane and is stabilized via the topological charge conservation.

Perhaps the deepest consequence of our new brane world is that it offers a natural explanation of the large mass hierarchy between the four-dimensional Planck scale $M_{\mathrm{Pl}}$ and the weak scale $m_{\mathrm{EW}}$. The large mass hierarchy is translated into the large size of the horizon. The large size of the horizon, i.e., the small compactification scale is provided with the large magnitude of charge carried by the black branes, that is, winding number. Hence, the macroscopic compactification size now is a quantity calculated from the microscopic theory and its stabilization is guaranteed from the charge conservation.

This picture also implies that one does not need to compactify all of the extra-dimensions to get the observed four-dimensional world. From an observer's viewpoint in the higherdimensional spacetime, near the horizon of the black branes, only the $(n-1)$ extra dimensions are compactified locally into $\mathbf{S}^{n-1}$ and the rest one extra dimension is warped. The apparent infinite extent of the near horizon space is simply a consequence of the warped region. Clearly, the interior region takes only a finite part of the higher-dimensional transverse space with volume $\sim r_{\mathrm{H}}^{n}$ and its apparent infinite extent is due to the warping by the gravity of the brane itself. Hence, the setup needed for a realistic brane world can be obtained simply from noncompact higher-dimensional spacetime by means of formation of such black-brane.

\section{Acknowledgement}

We are grateful to S. Dimopoulos, N. Kaloper and Choonkyu Lee for discussions, and I. Antoniadis and N. Arkani-Hamed for correspondences.

\section{References}

[1] N. Arkani-Hamed, S. Dimopoulos and G. Dvali, Phys. Lett. B429 (1998) 263; Phys. Rev. D59 (1999) 086004; 
[2] G. Shiu and S.-H.H. Tye, Phys. Rev. D58 (1998) 106007;

Z. Kakushadze and S.-H.H. Tye, Nucl. Phys. B546 (1999) 180;

L.E. Ibáñez, C. Muñoz and S. Rigolin, Nucl. Phys. B553 (1999) 43.

[3] I. Antoniadis and B. Pioline, Nucl. Phys. B550 (1999) 41;

K. Benakli, Phys. Rev. D60 (1999) 104002;

K. Benakli and Y. Oz, Phys. Lett. B472 (2000) 83.

[4] G. Giudice, R. Rattazzi and J. Wells, Nucl. Phys. B544 (1999) 3;

T. Han, J. Lykken and R. J. Zhang, Phys. Rev. D59 (1999) 105006.

[5] E. Mirabelli, M. Perelstein and M. Peskin, Phys. Rev. Lett. 82 (1999) 2236;

S. Nussinov, R. Shrock, Phys. Rev. D59 (1999) 105002;

C. Balasz et. al, Phys. Rev. Lett. 83 (1999) 2112;

J. L. Hewett, Phys. Rev. Lett. 82 (1999) 4765;

P. Mathew, K. Sridhar and S. Raichoudhuri, Phys. Lett. B450 (1999) 343;

T. G. Rizzo, Phys. Rev. D59 (1999) 115010;

K. Aghase and N. G. Deshpande, Phys. Lett. B456 (1999) 60;

K. Cheung and W. Y. Keung, Phys. Rev. D60 (1999) 112003;

L3 Coll. Phys. Lett. B464 (1999) 135.

[6] T.G. Rizzo, hep-ph/9910255

K. Cheung, hep-ph/0003306

I. Antoniadis and K. Benakli, hep-ph/0007226.

[7] D.H. Lyth, Phys. Lett. B448 (1999) 191;

N. Kaloper and A. Linde, Phys. Rev. D59 (1999) 101303;

N. Arkani-Hamed, et al., Nucl.Phys. B567 (2000) 189;

G. Dvali, S.H.H. Tye, Phys. Lett. B450 (1999) 72.

[8] I. Antoniadis and C. Bachas, Phys. Lett. B450 (1999) 83; N. Arkani-Hamed, L.J. Hall, D. Smith and N. Weiner, Phys. Rev. D62 (2000) 105002.

[9] L. Randall and R. Sundrum, Phys. Rev. Lett. 83, 3370 (1999).

[10] L. Randall and R. Sundrum, Phys. Rev. Lett. 83, 4690 (1999).

[11] N. Kaloper, Phys. Rev. D60 (1999) 123506; P. Kraus, J. High-Energy Phys. 9912 (1999) 011; M. Cvetic and J. Wang, hep-th/9912187. 
[12] C. Csaki, M. Graesser, C. Kolda and J. Terning, Phys. Lett. B462 (1999) 34;

H.B. Kim and H.D. Kim, Phys. Rev. D61 (2000) 064003;

P. Kanti, I. I. Kogan, K. A. Olive and M. Pospelov, Phys. Lett. B468 (1999) 31;

A. Kehagias and E. Kiritsis, J. High-Energy Phys. 9911 (1999) 022;

T. Shiromizu, K. Maeda and M. Sasaki, Phys. Rev. D62 (2000) 024012;

P. Binetruy, C. Deffayet, U. Ellwanger and D. Langlois, Phys. Lett. B477 (2000) 285;

E.E. Flanagan, S.H. Tye and I. Wasserman, Phys. Rev. D62 (2000) 044039;

C. Csaki, M. Graesser, L. Randall and J. Terning, Phys. Rev. D62 (2000) 045015;

J. E. Kim, B. Kyae, H. M. Lee, Nucl.Phys. B582 (2000) 296-312, (Erratum) ibid. B591 (2000) 587;

S. Mukohyama, T. Shiromizu and K. Maeda, Phys. Rev. D62 (2000) 024028.

[13] H. Verlinde, Nucl. Phys. B580 (2000) 264;

C. Csaki and Y. Shirman, Phys. Rev. D61 (2000) 024008;

A.E. Nelson, hep-th/9909001;

A. Chodos and E. Poppitz, Phys. Lett. B471 (1999) 119;

S.M. Carroll, S. Hellerman and M. Trodden, Phys. Rev. D62 (2000) 044049;

S. Nam, J. High-Energy Phys. 0003 (2000) 005;

N. Kaloper, Phys. Lett. B474 (2000) 269;

Z. Chacko and A. E. Nelson, Phys. Rev. D62 (2000) 085006.

[14] A. Brandhuber and K. Sfetsos, J. High-Energy Phys. 9910 (1999) 013.

[15] O. DeWolfe, D.Z. Freedman, S.S. Gubser and A. Karch, Phys. Rev. D62 (2000) 046008.

[16] M. Gremm, Phys. Lett. B478 (2000) 434.

[17] C. Grojean, J. Cline and G. Servant, Nucl. Phys. B578 (2000) 259;

D. Youm, Nucl.Phys. B576 (2000) 106;

M. Cvetic, H. Lu and C. N. Pope, hep-th/0001002;

K. Behrndt and S. Gukov, Nucl. Phys. B580 (2000) 225;

K. Behrndt and M. Cvetic, Phys. Lett. B475 (2000) 253;

K. Behrndt and M. Cvetic, Phys. Rev. D61 (2000) 101901;

M. Cvetic, H. Lu and C.N. Pope, hep-th/0001002;

M. Cvetic, H. Lu and C.N. Pope, hep-th/0002054:

M. Cvetic, H. Lu and C.N. Pope, hep-th/0007209;

M.J. Duff, J.T. Liu and K.S. Stelle, hep-th/0007120.

[18] R. Kallosh, A. Linde and M. Shmakova, J. High-Energy Phys. 9911 (1999) 010;

R. Kallosh and A. Linde, J. High-Energy Phys. 0002 (2000) 005. 
[19] H. Verlinde, hep-th/9908076;

E. Verlinde and H. Verlinde, J. High-Energy Phys. 0005 (2000) 034;

H. Verlinde, hep-th/0003236;

B.R. Greene, K. Schalm and G. Shiu, hep-th/0004103;

C.S. Chan, P.L. Paul and H. Verlinde, hep-th/0003236.

[20] N. Arkani-Hamed, S. Dimopoulos, G. Dvali and N. Kaloper, Phys. Rev. Lett. 84586 (2000).

[21] S. Forste, Z. Lalak, S. Lavignac, H.P. Nilles, Phys. Lett. B481 (2000) 360;

C. Grojean, J. Cline, G. Servant, Nucl. Phys. B578 (2000) 259;

S.P. de Alwis, A.T. Flournoy, N. Irges, hep-th/0004125

S.P. de Alwis, hep-th/0002174;

J.-W. Chen, M.A. Luty, E. Ponton, J. High-Energy Phys. 0009 (2000) 012;

V.A. Rubakov, Phys. Lett. B125 (1983) 139;

N. Arkani-Hamed, S. Dimopoulos, N. Kaloper, R. Sundrum, Phys. Lett. B480 (2000) 193;

S. Kachru, M. Schulz and E. Silverstein, Phys. Rev. D62 (2000) 085003;

C. Csaki, J. Erlich, C. Grojean, T. Hollowood, Nucl. Phys. B584 (2000) 359;

G.T. Horowitz, I. Low, A. Zee, Phys. Rev. D62 (2000) 086005;

Z. Kakushadze, Nucl. Phys. B589 (2000) 75;

Chuan-Jie Zhu, J. High-Energy Phys. 0006 (2000) 034;

S. Forste, Z. Lalak, S. Lavignac, H.P. Nilles, J. High-Energy Phys. 0009 (2000) 034;

V. Barger, T. Han, T. Li, J. D. Lykken, D. Marfatia, Phys.Lett. B488 (2000) 97;

P. Binetruy, J.M. Cline, C. Grojean, Phys. Lett. B489 (2000) 403;

H. Collins and B. Holdom, hep-th/0009127;

N. Tetradis, hep-th/0012106.

[22] A. G. Cohen and D. B. Kaplan, Phys. Lett. B470 (1999) 52.

[23] R. Gregory, Phys. Rev. Lett. 84 (2000) 2564.

[24] I. Olasagati and A. Vilenkin, Phys.Rev. D62 (2000) 044014.

[25] T. Gherghetta and M. Shaposhnikov, Phys. Rev. Lett. 85 (2000) 240.

[26] T. Gherghetta, E. Roessl and M. Shaposhnikov, Phys.Lett. B491 (2000) 353.

[27] I. Oda, hep-th/0006203.

[28] C. Csaki, J. Erlich, T. Hollowood and Y. Shirman, Nucl. Phys. B581 (2000) 309. 
[29] J. Lykken and L. Randall, J. High-Energy Phys. 0006 (2000) 014.

[30] J. Lykken, R. C. Myers and J. Wang, J. High-Energy Phys. 0009 (2000) 009.

[31] S.-H. Moon, in preparation.

[32] A. Chamblin and G. W. Gibbons, Phys. Rev. Lett. 84 (2000) 1090.

[33] D.J.H. Chung, E.W. Kolb and A. Riotto, hep-ph/0008126. 